\title{
Augmentation Characteristics and Microbial Community Dynamics of Low temperature Resistant Composite Strains LTF-27
}

Guoxiang Zheng ( $\nabla$ zgx720331@126.com )

Northeast Agricultural University

Stopira Yannick Benz Boboua

Northeast Agricultural University

Chenyang Zhou

Northeast Agricultural University

Jiachen Li

Northeast Agricultural University

Weishuai Bi

Northeast Agricultural University

Ruxian Wang

Northeast Agricultural University

Shengnan Chen

Northeast Agricultural University

\section{Research Article}

Keywords: augmentation characteristics, microbial community dynamics, psychrotrophic, lignocellulosedegrading consortium LTF-27, anaerobic digestion

Posted Date: September 10th, 2021

DOl: https://doi.org/10.21203/rs.3.rs-875071/v1

License: (c) (i) This work is licensed under a Creative Commons Attribution 4.0 International License. Read Full License 


\section{Abstract}

Biogas production in the cold regions of China is hindered by low temperatures, which led to slow lignocellulose biotransformation. Cold-adapted lignocellulose degrading microbial complex community LTF-27 was used to investigate the influence of hydrolysis on biogas production. After 5 days of hydrolysis at $15 \pm 1^{\circ} \mathrm{C}$, the hydrolysis conversion rate of the straw went up to $22.64 \%$, and the concentration of acetic acid rose to $2,596.56 \mathrm{mg} / \mathrm{L}$. The methane production rates of TS inoculated by LTF-27 reached 204.72 $\mathrm{ml} / \mathrm{g}$, which was higher than the biogas $(161.34 \mathrm{ml} / \mathrm{g})$, and the CK $(121.19 \mathrm{ml} / \mathrm{g})$, the methane production rate of VS increased by $26.88 \%$ and $68.92 \%$, respectively. Parabacteroides, Lysinibacillus, and Citrobacter were the main organisms that were responsible for hydrolysis. While numerous other bacteria genera in the gas-producing phase, Macellibacteroides were the most commonly occurring one. Methanosarcina and Methanobacteriaceae contributed $86.25 \%$ and $11.80 \%$ of the total Archaea abundance during this phase. This study proves the psychrotrophic LTF-27's applicability in hydrolysis and biomass gas production in low temperatures.

\section{Introduction}

In recent years, due to the excessive development and the fast depletion of fossil fuel reserves, the corresponding environmental pollution has also become a serious and irreversible environmental problem (Mei et al. 2017). Therefore, research for new renewable and environmentally friendly energy sources to replace the overexploitation of traditional energy sources has become the focus of global attention today (Zheng et al. 2020). Crop straws (CS), which have excellent biomass qualities, are a vital biomass resource that, if used well, can relieve the pressure of global climate change (Wang et al. 2019a). Anaerobic digestion (AD) plays a important role in sustainable development by transforming organic wastes into green energy (Peu et al. 2017). Bioenergy, especially biogas produced through the AD of renewable feedstock, is considered one of the up-and-coming alternatives to fossil-derived energy due to several inherent and significant merits (Cheng et al. 2011; Kaparaju et al. 2009). As an efficient process for CS treatment, biogas $A D$ has excellent potential for application prospects. Firstly, it could effectively degrade $C S$ and reduced its impact on the environment. Secondly, compared to the aerobic fermentation treatment process, the construction and design cost of the $A D$ treatment process was lower, and the energy consumption was relatively lower (Zhang et al. 2018).

Furthermore, the $A D$ was a complex multi-step microbial process, and the synergy between these microbial floras was a prerequisite to ensure the system's stable operation (Ward et al., 2017). To enhance the biological activity of the $A D$ system and accelerate the reaction rate, bioaugmentation was also widely adopted, particularly in the hydrolysis stage, is expected to result in enhanced biogas production rates owing to sophisticated enzyme systems produced anaerobic hydrolytic bacteria, including the Firmicutes, Bacteroidetes, Fibrobacter, Spirochaetes, and Thermotogae (Kinet et al. 2015; Peng et al. 2014; Azman et al. 2015). It is worth quoting that $A D$ systems inoculated with the thermophilic or mesophilic microbial community have better performance due to improving acidogenesis due to improved methane yield in biogas production systems in succession (Ozbayram et al. 2017; Jin et al. 2018). But, nowadays, there are fewer reports for that. 
It is well known that the properties of the straw made it difficult for the microorganism to adapt and rendered the straw susceptible to spoilage at low temperatures (Chen et al. 2017; Wang et al. 2019; Sun et al. 2017). Therefore, bioaugmentation by the inoculated psychrotrophic lignocellulose-degrading consortium was significant in accelerating bioconversion of lignocellulosic wastes in straw-methane production engineering in northeast cold regions China. In this study, the low-temperature resistant microbial consortium LTF-27 screened by the Biomass Energy Research Center of Northeast Agricultural University (Harbin, China) (Zheng et al. 2020) was used to augmenting the hydrolysis phase in two-phase of AD. It accelerated the reaction rate in the hydrolysis phase and increase the rate of biogas production. By comparing the hydrolysis effect of the CK and the blank control group using the biogas's suspension for hydrolysis, the promotion effect of the complex strain on the fermentation system was explored. The changes in the microbial population structure at different stages of the AD process were monitored to explore the mechanism of action of microorganisms during the AD process. This study proves the psychrotrophic LTF-27's characteristics and applicability in hydrolysis and biomass gas production in low temperatures.

\section{Materials And Methods}

\subsection{Materials}

The LTF-27 microbial community was isolated through serial subcultivation at a low temperature of $15 \pm$ $1^{\circ} \mathrm{C}$ under static facultative anaerobic conditions. There were efficacious inocula kept at $-80^{\circ} \mathrm{C}$ with $80 \%$ glycerol as carriers for the inoculation (Zheng et al. 2020). The medium of microbial consortium LTF-27 used a peptone cellulose medium (PCS) (Fang et al. 2018). The specific components were: peptone $5.0 \mathrm{~g}$, cellulose $5.0 \mathrm{~g}, \mathrm{NaCl} 5.0 \mathrm{~g}, \mathrm{CaCO}_{3} 2.0 \mathrm{~g}$, yeast powder $1.0 \mathrm{~g}$, and dissolved in $1 \mathrm{~L}$ of distilled water; the $\mathrm{pH}$ was natural to set at $\mathrm{pH} 7.0$.

The experimental raw materials used were corn stalk pretreated by using the $\mathrm{NaOH}$ method; according to Zheng et al. (2020), cow manure (CM) was collected from a farm of Northeast Agricultural University (Harbin, China). After naturally air-drying, the corn stalk was sprayed in 2-3 $\mathrm{mm}$ with a sprayer. The basic parameters of the test materials were shown in Table 1).

Table 1

Physical and chemical properties of test materials

\begin{tabular}{|lclllllll|}
\hline Materials & $\mathbf{C ( \% )}$ & $\begin{array}{l}\mathbf{N} \\
(\%)\end{array}$ & $\mathbf{C / N}$ & TS(\%) & VS(\%) & Cellulose(\%) & Hemicellulose(\%) & Lignin(\%) \\
\hline $\begin{array}{l}\text { Corn } \\
\text { stalks }\end{array}$ & 31.68 & 0.44 & 65 & 91.24 & 87.13 & 36.67 & 25.82 & 8.78 \\
\hline Biogas & 25.26 & 1.32 & 19.14 & 5.42 & 4.18 & - & - & - \\
\hline
\end{tabular}


In this study, the low temperature-resistant microbial consortium LTF-27 was used as an intensive enhanced strain in the hydrolysis and acid production phase, in which the temperature was set at $15 \pm 1^{\circ} \mathrm{C}$. The reaction mixture was hydrolysis in an apparatus at stage $1 \mathrm{~L}$ Erlenmeyer flask, in which the corn straws and the PCS culture solution were added to it. The practical volume of the reaction was $0.7 \mathrm{~L}$. The concentration of the hydrolysis substrate in the control system was $8 \%$, the initial pH value and the inoculum of LTF-27 were 7.0 and $27 \%$, respectively. After sterilizing and inoculating with low-temperature resistant novel microbial consortium LTF-27, hydrolysis was set at 5 days and regularly daily sampling. The indicators of the hydrolysis phase were measured. The sample was obtained at the beginning of each cycle to determine the chemical composition of the reactor and microbial composition (Liu et al. 2018). At the end of the hydrolysis phase, the straw from the hydrolyzed phase and the acidified liquid was transferred into $2 \mathrm{~L}$ wide-mouth flack inoculated with the domesticated methane solution; the ratio of acidification liquid and domesticated methane solution was 1:1. The follow-up of anaerobic gas production was carried out at $35 \pm$ $1^{\circ} \mathrm{C}$, in which the straw hydrolyzed and fermented the liquid from the hydrolysis phase was used further to produce acid for subsequent anaerobic gas production. The gas production and methane content of the gas-producing phase were regularly monitored (Wu et al. 2017).

\subsection{Analytical Methods}

According to the techniques described by the Von Soest method, a fiber analyzer (Model ANKOM220, USA) was used to determine lignin, cellulose, and hemicellulose (Chu et al. 2020; Liu et al. 2018). The total solid content (TS) and the volatile solids content (VS) were measured using the national standard method (Yin et al. 2016). Samples for VFAs analysis were first centrifuged at 10,000 for 5 minutes, the supernatants were then filtered through a $0.22 \mu \mathrm{m}$ filter, and filtrates were collected in sample vials for analysis (Liu et al. 2018; Mirdamadi et al. 2015). The content of VFAs and biogas were determined using an Agilent $6890 \mathrm{~N}$ of the type determined by gas Chromatography (Zheng et al. 2020). The VAFs yields were calculated as the cumulative sum of the measured acetic ( $\mathrm{Hac})$, propionic ( $\mathrm{HPr})$, etc. The daily gas production in the gas production stage was measured by the drainage method, and the gas composition in the biogas was measured by an Agilent 7890N gas chromatograph (Lin et al. 2016; Sun et al. 2020).

The scanning electron microscope was used to observe and record the morphological properties of the microbial flora. The genomic DNA was extracted using Ezup Column Soil DNA Purification Kit (Shanghai Biotech, China). The V3-V4 region of 16S rRNA genes of bacteria and archaea were amplified according to the sequences of the primers presented in Table 2. The polymerase chain reaction (PCR) was performed (Liu et al. 2018). Major Bio-Pharm Biotechnology Co., Ltd. (Shanghai, China) used an Illumina Miseq PE250 to perform high-throughput pyrosequencing on purified PCR products. The raw reads were deposited into the NCBI Sequence Read Archive (SRA) database (Shi et al. 2017). Shanghai Major Bio-Pharm Technology Co., Ltd. (Shanghai, China) was responsible for analyzing these data. 
Table 2

the microeconomic primer sequences

\begin{tabular}{|lll|}
\hline Item & Primer & Sequence \\
\hline Bacteria & $341 \mathrm{~F}$ & CCCTACACGACGCTCTTCCGATCTG \\
& $850 \mathrm{R}$ & GACTGGAGTTCCTTGGCACCCGAGAATTCCA \\
Archaea & $349 \mathrm{~F}$ & CCCTACACGACGCTCTTCCGATCTN \\
& $806 \mathrm{R}$ & GACTGGAGTTCCTTGGCACCCGAGAATTCCAGGACTACVSGGGTATCTAAT \\
\hline
\end{tabular}

\subsection{Statistical analysis}

SPSS 20 and ORIGIN pro 8.0 were used to process the data collected at the genus level of microbial communities and degradation rates in different phase parameters.

\section{Results And Discussion}

\subsection{Effect of inoculation with microbial consortium LTF-27 on the hydrolysis effect of the system}

\subsubsection{The degradation of straw and its component}

The straw degradation effect at the end of each test group was shown in (Fig. 1). In the low-temperature environment $\left(15^{\circ} \mathrm{C}\right)$, the degradation efficiency of the straw in the control group (CK) was very low in the first five days of the hydrolysis phase. The straw degradation rate was $3.61 \%$, which the degradation rate of cellulose, hemicellulose, and lignin was $2.38 \%, 4.13 \%$, and $0.4 \%$, respectively. On the other hand, all indicators of the experimental group inoculated with the microbial consortium LTF-27 were significantly improved. At the end of the hydrolysis phase, the degradation rate of straw reached $22.64 \%$, which of the degradation rate of cellulose, hemicellulose, and lignin was $23.58 \%, 27.14 \%$, and $4.58 \%$, respectively. Cellulose and hemicellulose were relatively easily degraded compared to lignin (Wang et al. 2019b). The straw decomposed rate inoculated with biogas was $14.58 \%$. The degradation rates of cellulose and hemicellulose were $15.31 \%$ and $18.63 \%$, respectively, which were lower than the degradation rate of the test group using the composite strain LTF-27. These results indicate that LTF-27 can effectively improve the biodegradability of complex substrates (Zheng et al. 2020). The microbial flora was more suitable adapted to acid production by hydrolysis. At the same time, the microorganisms in the biogas suspension were relatively complicated, and the rate of acid production by hydrolysis was lower than that of the microbial consortium LTF-27. It could be seen that the microbial consortium LTF-27 had a high degradation capacity and could degrade straw in a low-temperature environment, thus improving the raw material utilization efficiency and reaction speed of the system.

\subsubsection{The production of primary liquid end product acetic acid}


As the main product of the hydrolysis process, the acetic acid content directly determined the efficiency of the gas production stage in the system (Wang et al. 2019). There was a higher concentration of acetic acid in the hydrolysis process. In the early research, Wang et al. (2013) reported that acetic acid and butyric acid were the primary significant organic acids beneficial for subsequent methane fermentation in the $A D$ process. More substrates are available for the gas-producing phase of the methanogens in that gas production stage, which could accelerate the growth and metabolism rate of methanogens, thereby improving the gas production efficiency in the system. The variation in acetic acid content during the hydrolysis process was illustrated in (Fig. 2). Although the acetic acid concentration increased slightly in the $\mathrm{CK}$ and was always low, $457.68 \mathrm{mg} / \mathrm{L}(\mathrm{p}<0.05)$ at the low-temperature $15 \pm 1^{\circ} \mathrm{C}$. The concentration of acetic acid increased during the hydrolysis process in the test group inoculated with biogas. It reached $1,607.32 \mathrm{mg} / \mathrm{L}$ in the first five days, lower than the test group inoculated with complex strains LTF-27 $(2,596.56 \mathrm{mg} / \mathrm{L})$. Because there were so many microorganisms in biogas, acid-producing microorganisms must compete with other flora for nutrients and influence the VFA production yield. Compared to the last two groups, the test group inoculated with microbial consortium LTF-27 had an acetic acid concentration of $456.84 \mathrm{mg} / \mathrm{L}$ in 1 day, then increased rapidly during the hydrolysis and acidification process. It reached $2,956.56 \mathrm{mg} / \mathrm{L}$ at 5 days, indicating that the microbial flora grew rapidly and reproduced at a rapid rate during the hydrolysis process, making good used of the nutrients in the system compared to the previous two groups. The addition of microbial consortium LTF-27 was conducted to hydrolysis straw to produce acetic acid, which was effectively beneficial for subsequent methane fermentation in the AD process (Wang et al. 2019).

\subsubsection{The biogas production and methane yield}

The cumulative gas production of each test group was shown in (Fig. 3). The increase in the cumulative gas production rate of the test group inoculated with the microbial consortium LTF-27 increased rapidly, mainly due to the better straw substrate degradation in the hydrolysis process, and the better acetic acid production in the system during the hydrolysis process, and more substrates for the methanogenic bacteria to use in the gas production process, resulting in higher gas production efficiency. The total gas production of the experimental group inoculated with the microbial consortium LTF-27 reached 18,110 ml in 25 days. The total gas production of the experimental group inoculated with biogas was $14,890 \mathrm{ml}$, and the total gas production of the control group was $11,170 \mathrm{ml}$. it could be seen that the acidification effects in the hydrolysis stage would directly affect the gas production rate. The gas production affected the system's volume. In this study, the inoculation of microbial consortium LTF-27 could effectively enhance the hydrolysis and acidification effect, thus improving the gas production effect of the whole system.

Methane is the main component of biogas, and the methane content level directly determines the biogas quality. Methane yield can visually reflect the methane production of a system (Dichter et al. 2020). The methane production rates of TS and VS in each test group were shown in (Fig. 4). Methane production rates of TS and VS in the test group inoculated with LTF-27 were higher than the other two groups, with the methane production rate of TS and VS reaching $193.17 \mathrm{ml} / \mathrm{g}$ of TS and $204.72 \mathrm{ml} / \mathrm{g}$, respectively. Methane production rates of TS and VS in the test group inoculated with biogas were $152.28 \mathrm{ml} / \mathrm{g}$ and $161.34 \mathrm{ml} / \mathrm{g}$, respectively. 
In contrast, the methane production rates of TS and VS in the control group were $116.84 \mathrm{ml} / \mathrm{g}$ and 121.19 $\mathrm{ml} / \mathrm{g}$, respectively. Methane production rate of TS in the test group inoculated with LTF-27 increased by $65.33 \%$ compared with the control group CK, and by $26.85 \%$ in the test group inoculated with biogas compared with the control group CK. Besides, the methane production rate of VS in comparison increased by $68.92 \%$ and $26.88 \%$, respectively. Thus, it could be seen that inoculation of the low temperature tolerant microbial consortium LTF-27 could significantly improve the hydrolysis effect of the system during the hydrolysis process and then improved the methane yield of the system.

\subsection{The morphological characteristics of hydrolysis ans gas- producing phase}

This study analyzed only the microbial community structure of the acid-producing and gas-producing phases inoculated with LTF-27.

The changes of microbial morphological characteristics at different stages of two-phase anaerobic digestion were observed using electron scanning electron microscopy, as shown in (Fig. 5). In the prehydrolysis phase, the number of microorganisms in the system was insignificant, and the colonies were scattered. There were mainly rod-shaped and globular bacteria in the system, indicating more types of microorganisms in the initial hydrolysis stage. After a period of growth and reproduction, straw can be used as a carbon source. The dominant flora of growth was more obvious. In the middle of hydrolysis, the microbial flora was denser than the initial stage, which indicated that the activity of the flora was enhanced and could grow and metabolize normally. Bacilli dominated the flora in the system, and the number of cocci was reduced, indicating that Bacilli could better adapt to growth in the hydrolysis stage and were the dominant flora in the system (Zhang et al. 2020). In the last hydrolysis stage, the microbial flora was denser, and most of the flora in the system was more stable and metabolized normally. The system was mainly composed of Bacilli, proving that the dominant flora in the hydrolysis stage was Bacillus. The environment temperature was appropriate for microbial growth in the hydrolysis stage (Sun et al. 2017).

In the gas production phase (see Fig. 5), the community structure of the microorganisms in the system changed due to the addition of methane into the system. In the initial stage of gas production (sampling was taken on the 1st day of gas production), there were many microorganisms in the system. Various microorganisms were growing and multiplying, and then the microorganisms suitable for the environment were growing normally. The microorganisms that could not use the substances in the system gradually disappeared. In the middle stage of gas production (sampling was taken on the 10th day of gas production), the morphology of bacterial flora was mainly spherical and rod-shaped. Combined with the analysis of gas production, it showed that most of these microorganisms were using the substrates in the system to convert to produce methane, carbon dioxide $\left(\mathrm{CO}_{2}\right)$, and other gases. Compared with the previous research, the gram-positive, rod-shaped bacteria utilized $\mathrm{CO}_{2}$ as a source of energy (sampling 23 energy sources to reduce methanol to $\mathrm{CH}_{4}$ ) (Kumar et al. 2018). The specific classification of microorganisms should be obtained by molecular sequencing. The last stage of gas production ( sampling was taken on the 20th day of gas production), as most of the substrates in the system, were used and the nutrients were gradually depleted, resulting in the reduction of microbial activity and the change of microbial community 
structure accordingly, and morphologically, the last stage of gas production microbial community was mainly rod-shaped bacteria. In conclusion, anaerobic digestion was a complex biological process, and each stage required corresponding microbial populations to complete in the concert of it. As a result, the dominant microbial flora at the hydrolysis stage and the gas production stage was very different, and the microbial population in the system was affluent (Sun et al. 2017).

\subsection{Microbial community structure and dynamics during fermentation}

The alpha-diversity indices for each sample group could be seen in Table 3. The coverage index represented the authenticity of the samples, and the library coverage of each sample group was above $97 \%$, indicating that the sample detection rate was high and the results were reliable (Li et al. 2016; Werner et al. 2011). The Ace and Chao indices were used to reflect the overall number of species in the sample. During the hydrolysis phase, the S1-2 group had a more significant total number of species. The Ace index was $39,660.66$, and the Chao index was $14,347.125$, indicating that microorganisms multiplied and grew in the system during the last hydrolysis phase. The total number of microorganisms was huge. The Ace and Chao indices of group S1-1 were minimal. The values of 13,087.86 and 8,483.64, respectively, indicated that the total number of microorganisms in the system at the initial hydrolysis phase was not significant. Each microbial flora should grow and multiply. The Shannon and Simpson indexes reflected the diversity of microorganism samples. The group with higher microbial diversity during the hydrolysis phase was the S11 group. The Shannon index was 5.31 , and the Simpson index was 0.02 , indicating that the species of microbial flora in the initial hydrolysis system was the most important. In the last hydrolysis stage, the number of microorganisms was low. Combined with the total amount of microorganism numbers, it could be seen that there were many types of microorganisms in the system at the initial phase of the hydrolysis. After growth and reproduction, some of the flora were not adapted to the growth of the system's environment and gradually disappeared (Werner et al. 2011). The medium-growing and metabolizing flora could use the nutrients in the system to grow and reproduce continuously, and the total number of flora became large. As a result, the Shannon and Simpson index decreased, and the Ace and Chao index increased. In the gas-producing phase, the sample of the Ace index reached $71,630.63$, and the Chao index reached $24,684.9$, which was higher than the value of the hydrolysis phase, indicating that the total number of microorganisms in the system during the gas-producing phase was very high. While the Shannon index was 1.58 and the Simpson index was 0.32 , indicating that the microbial diversity in the system was relatively high and the flora was vibrant. The growth and metabolism of each flora might be better, and the system was functioning stably. The Shannon index was 1.25 in the gas-producing phase, which was lower than the diversity index of F1-1 bacteria (1.58), indicating that the diversity of bacteria in the sample was higher than that of the archebacteria. 
Table 3

Statistical table of bacterial Alpha diversity in each group of samples

\begin{tabular}{|llllllll|}
\hline $\begin{array}{l}\text { Sample } \\
\text { ID }\end{array}$ & $\begin{array}{l}\text { Seq } \\
\text { num }\end{array}$ & $\begin{array}{l}\text { OTU } \\
\text { num }\end{array}$ & $\begin{array}{l}\text { Shannon } \\
\text { Index }\end{array}$ & $\begin{array}{l}\text { Ace } \\
\text { Index }\end{array}$ & $\begin{array}{l}\text { Chao } \\
\text { Index }\end{array}$ & $\begin{array}{l}\text { Coverage } \\
\text { Index }\end{array}$ & $\begin{array}{l}\text { Simpson } \\
\text { Index }\end{array}$ \\
\hline S1-1 & 63,968 & 2,817 & 5.31 & $13,087.86$ & $8,483.64$ & 0.97 & 0.02 \\
\hline S1-2 & 42,111 & 714 & 1.22 & $39,660.66$ & 14347.125 & 0.98 & 0.48 \\
\hline F1-1 & 65,758 & 1,041 & 1.58 & $71,630.63$ & $24,684.9$ & 0.99 & 0.32 \\
\hline F1-1 & 22,983 & 600 & 1.25 & $17,155.37$ & $9,098.81$ & 0.98 & 0.65 \\
(archaea) & & & & & & & \\
\hline
\end{tabular}

The distribution of the microbial flora of the genus in the experiment group inoculated LTF-12 during the hydrolysis stage was illustrated in (Fig. 6). The relatively abundant flora was Parabacteroides, Lysinibacillus, and Citrobacter. In the pre-hydrolysis phase (Fig. 6-A), the maximum relative abundance of the genus Parabacteroides in the system was $66.49 \%$, followed by Lysinibacillus (15.58\%), Citrobacter $(11.53 \%)$, and the total flora content of these three bacteria reached the overall $93.60 \%$. At the end of the hydrolysis phase, the dominant flora in the rest of the system was still Parabacteroides, Lysinibacillus, and Citrobacter. Then, however, the relative abundance changed considerably, and the content of Lysinibacillus and Citrobacter increased to $25.49 \%$ and $19.37 \%$, respectively. In comparison, the relative abundance of Parabacteroides decreased to $46.12 \%$ but remained the most abundant floras in the system (Fig. 6-B). Thus, we could see that Lysinibacillus and Citrobacter could adapt to the system environment, multiply in large quantities, and better degrade straw.

Compared with the composition of the community of microbial consortium LTF-27 seen from (Fig. 7-A) (Zheng et al. 2020), it could be seen that the content of Parabacteroides (66.49\%) was most abundant in the microbial consortium LTF-27, which could also be adapted to the growth and metabolism of straw as a source of carbon production, and adapted to complete the task of hydrolytic acid production. The relative abundance of Lysinibacillus (15.58\%) in the system was significantly improved, indicating that this strain could be better utilized for the nutrients contained in the straw. The most significant increase in relative abundance was Citrobacter(11.53\%). This genus Citrobacter could adapt to the system environment and grow and reproduce in large numbers (Zheng et al. 2020) decompose straw in the system to produce acid.

Parabacteroides were an essential member of heterophils involved in organic carbon cycling and proteinaceous substances (Kokkwang et al. 2016; Shi et al. 2015). Moreover, the studies reported that the hydrolysis of biomasses was positively correlated with the percentage of Parabacteroides (Regueiro et al. 2012). The genus Lysinibacillus was gram-positive and had perennial flagella. Most of them specialized in anaerobic bacteria to degrade large molecule organic compounds such as proteins, carbohydrates, and cellulose, and some of the genus Lysinibacillus also had nitrogen fixation effect (Liu et al. 2019; Roy et al. 2014; Roy et al. 2016). 
Due to biogas suspension in the system during the gas production process, the number of microorganisms was diverse and complicated (Fig. 8-A), consistent with the previous reports (Zheng et al. 2020). There were about $36.62 \%$ non-class bacteria presented in the methane phase due to the limitation of the database in bacteria-genus level, shown in Fig. 7-A, the most abundant identified were Macellibacteroides with a relative abundance of (7.98\%), followed by Clostridium III with (4.39\%), Ruminococcus (3.48\%), Parabacteroides (3.34\%), and Lysinibacillus (3.19\%). At the archaea-genus level, methanogens were the absolute dominant flora group of the Archean community in the methanogenic phase, as illustrated in (Fig. 8-B). The relative abundance of Methanosarcina was the highest, reaching $85.25 \%$, which was the optimal flora in the gasproducing phase system. The cell morphology of Methanosarcina was spherical asymmetrical, and the optimal temperature for the growth of mesophilic bacteria was $30^{\circ} \mathrm{C}-40^{\circ} \mathrm{C}$ (Ge et al. 2014). It was the only Archaea capable of forming a cell structure using extracellular polysaccharides. Methanosarcina generally used $\mathrm{H}_{2} / \mathrm{CO}_{2}$, methanol, acetic acid, methylamine, and other substances to generate biogas, and could enhance the performance of the AD reactor and improve the system's stability (Peu et al. 2017). Methanobrevibacter content was also very high, with a relative abundance of $11.80 \%$, seen in (Fig. 8-B). Methanobrevibacter was often present in $A D$ reactors, animal rumen, and anaerobic activated sludge, which was strictly anaerobic, using mainly $\mathrm{H}_{2} / \mathrm{CO}_{2}$ and formic acid as energy sources to produce $\mathrm{CH}_{4}$ (Kumar et al. 2018). Methanosarcina and Methanobacteriaceae accounted for about $97.05 \%$ of the total quantity of archaea and jointly converted substances in the gas production system to methane (Hosur et al. 2020). The previous research demonstrated the archaea consisting of acetoclastic methanogens of the family Methanosaetaceae remained stable (Kumar et al. 2018). Our studies also confirmed that the gas-producing phase was a series of biological reactions directed by methanogens. The methanogen $s$ and acid-producing bacteria acted in concert and maintained the stable operation of anaerobic gas production.

\section{Conclusion}

The psychrotrophic lignocellulose-degrading microbial consortium LTF-27 could make the straw get effective degradation, release the feedback inhibition of harmful the buildup of metabolites, and realize the efficient degradation of lignocellulosic substances at $15 \pm 1^{\circ} \mathrm{C}$. Therefore, LTF-27 could be suitable for augmenting treatment from lignocellulose wastes hydrolysis and increase methane production effectively, and demonstrating that it could be applied to the improved energy balance for methane production at low temperatures. These results should have significant economic implications, and increasing the lignocellulose treatment strategy's potential for large-scale straw-methane fermentation engineering in the cold regions of northeast China.

\section{Declarations}

\section{Acknowledgments}

The financial research support received from the National Key R\&D Program of China (2019YFD1100603), National Key Research and Development Program Strategic Key Special Subject of International Science 
and Technology Innovation Cooperation (2018YFE026602) and National Natural Science Foundation of China (52076034) is gratefully acknowledged.

\section{References}

Amani T, Nosrati M, Sreekrishna TR (2010) Anaerobic digestion from the viewpoint of microbiological, chemical, and operational aspects - a review. Environ Rev 18: 255-278. https://doi.org/10.1139/A10-011

Chen H, Liu J, Chang X, Chen D, Xue Y, Liu P, Lin H, Han S (2017) A review on the pretreatment of lignocellulose for high-value chemicals. Fuel Processing Technol. 160: 196-206.

https://doi.org/10.1016/j.fuproc.2016.12.007

Cheng CL, Lo YC, Lee KS, Lee DJ, Lin CY, Chang JS (2011) Biohydrogen production from lignocellulosic feedstock. Bioresour. Technol. 102: 8514-8523. https://doi.org/10.1016/j.biortech.2011.04.059

Chu X, Kumar Awasthi M, Liu Y, Cheng Q, Qu J, Sun Y (2020) Studies on the degradation of corn straw by combined bacterial cultures. Bioresour. Technol. 320, 124174.

https://doi.org/10.1016/j.biortech.2020.124174.

Dichter N, Aboud A (2020) Analysis of Greenhouse Gas Emissions from Residential Heating Technologies in the USA. UC Davis Western Cooling Efficiency Center. https://wcec.ucdavis.edu/wp-content/uploads/GHGEmissions-from-ResidentialHeating-Technologies-091520.pdf

Fang X, Li Q, Lin Y, Lin X, Dai Y, Guo Z, Pan D (2018) Screening of a microbial consortium for selective degradation of lignin from tree trimmings. Bioresour. Technol. 254: 247-255.

https://doi.org/10.1016/j.biortech.2018.01.058

Ge X, Yang L, Sheets JP, Yu Z, Li Y (2014). Biological conversion of methane to liquid fuels: status and opportunities. Biotechnol. Adv. 32: 1460-1475. https://doi.org/10.1016/j.biotechadv.2014.09.004

Hosur KH, Betha UK, Yadav KK, Mekapogu M, Kashyap BK (2020) Byproduct Valorization of Vegetable Oil Industry Through Biotechnological Approach. In: Kashyap BK, Solanki MK, Kamboj DV, Pandey AK (eds) Waste to Energy: Prospects and Applications. Springer, Singapore. https://doi.org/10.1007/978-981-334347-4_8

Hung $\mathrm{C} \mathrm{H}$, Chang YT, Chang YJ (2011) Roles of microorganisms other than Clostridium and Enterobacter in anaerobic fermentative biohydrogen production systems-a review. Bioresour. Technol. 102: 8437-8444. https://doi.org/10.1016/j.biortech.2011.02.084

Ji J, Kakade A, Yu Z, Khan A, Liu P, Li X (2020) Anaerobic membrane bioreactors for treatment of emerging contaminants: A review. J. of Environ. Management. 270:

110913. https://doi.org/10.1016/j.jenvman.2020.110913 
Jin W, Xu X, Yang F, Li C, Zhou M (2018) Performance enhancement by rumen cultures in anaerobic codigestion of corn straw with pig manure. Biomass and Bioenergy. 115: 120-129. https://doi.org/10.1016/j.biombioe.2018.05.001

Kaparaju P, Serrano M, Thomsen AB, Kongjan P, Angelidaki I (2009) Bioethanol, biohydrogen and biogas production from wheat straw in a biorefinery concept. Bioresour. Technol. 100: 2562-2568. https://doi.org/10.1016/j.biortech.2008.11.011

Kokkwang N, Shi XQ, Sayleong O, Howyong N (2016) Pyrosequencing reveals microbial community profile in anaerobic bio-entrapped membrane reactor for pharmaceutical wastewater treatment. J. Bioresour. Technol. 200: 1076-1079. https://doi.org/10.1016/j.biortech.2015.10.100

Kumar S, Hans M (2018) Biohythane production in two-stage anaerobic digestion system. J. Hydrogen Energy 44(32): 17363-17380. https://doi.org/10.1016/j.ijhydene.2018.10.022

Lin Q, Vrieze JD, Li JB, Li XZ (2016) Temperature affects microbial abundance, activity and interactions in anaerobic digection. Bioresour. Technol. 209: 228- 236. https://doi.org/10.1016/j.biortech.2016.02.132

Liu CM, Wachemo AC, Tong H, Shi SH, Zhang L, Yuan HR, Li XJ (2018) Biogas production and microbial community properties during anaerobic digestion of corn stover at different temperatures.

Bioresour.Technol. 261: 93-103. https://doi.org/10.1016/j.biortech.2017.12.076

Liu M, Han Z, Yang Y (2019) Accumulation, temporal variation, source apportionment and risk assessment of heavy metals in agricultural soils from the middle reaches of Fenhe River basin. J. North China. RSC Advances. 9 (38): 21893-21902. https://doi.org/10.1039/C9RA03479J

Liu Z, Si B, Li J, He J, Zhang C, Lu Y, Zhang Y, Xing X.H (2018) Bioprocess engineering for biohythane production from low-grade waste biomass: technical challenges towards scale up. J. Curr. Opin. Biotechnol. 50: 25-31. https://doi.org/10.1016/j.copbio.2017.08.014

Mei X, Xing D, Yang Y, Liu Q, Zhou H, Guo C, Ren N (2017) Adaptation of microbial community of the anode biofilm in microbial fuel cells to temperature. J. Bioelectrochemistry. 117: 29-33.

https://doi.org/10.1016/j.bioelechem.2017.04.005

Mirdamadi S, Ghazvini SA (2015) A comparative study between inhibitory effect of L. lactis and nisin on important pathogenic bacteria in Iranian UF Feta cheese. Biological J. of Microorganism. 3: 79- 92

Ozbayram EG, Kleinsteuber S, Nikolausz M, Ince B, Ince O (2017) Effect of bioaugmentation by cellulolytic bacteria enriched from sheep rumen on methane production from wheat straw. Anaerobe. 46: 122-130. https://doi.org/10.1016/j.anaerobe.2017.03.013

Peng XW, Börner RA, Nges, IA (2014) Impact of bioaugmentation on biochemical methane potential for wheat straw with addition of Clostridium cellulolyticum. Bioresour. Technol. 152: 567-571. https://doi.org/10.1016/j.biortech.2013.11.067

Page 12/20 
Peu P, Tian JH, Pourcher AM, Bureau C (2017) Cellulose accessibility and microbial community in solid state anaerobic digestion of rape straw. Bioresour. Technol. 223: 192-201.

https://doi.org/10.1016/j.biortech.2016.10.009

Regueiro L, Veiga P, Figueroa M, Alonso-Gutierrez J, Stams AJ, Caballa M (2012) Relatonship between microbial activity and microbial community structure in six full-scale anaerobic digesters. J. Microbiol. Res. 167: 581-589. https://doi.org/10.1016/j.micres.2012.06.002

Roy S, Das D (2016) Biohythane production from organic wastes: present state of art. J. Environ. Sci. and Pollution Research. 23: 9391-9410. https://doi.org/10.1007/s11356-015-5469-4

Roy S, Vishnuvardhan M, Das D (2014) Continuous thermophilic biohydrogen production in packed bed reactor. J. Appl. Energy. 136: 51-58. https://doi.org/10.1016/j.apenergy.2014.08.031

Sawatdeenarunat C, Surendra KC, Takara D, Oechsner H, Kumar Khanal S (2015) Anaerobic digestion of lignocellulosic biomass: challenges and opportunities. Bioresour. Technol. 178: 178-186.

https://doi.org/10.1016/j.biortech.2014.09.103

Shi X, Ng KK, Li XR, Ng HY (2015) Investigation of intertidal wetland sediment as a novelinoculation source for anaerobic saline wastewater treatement. J. Environ. Sci. Technol. 49: 6231-6239.

https://doi.org/10.1021/acs.est.5b00546

Sun Q, Wu D, Zhang Z, Zhao Y, Xie X, Wu J, Lu Q, Wei Z (2017) Effect of cold-adapted microbial agent inoculation on enzyme activities during composting start-up at low temperature. Bioresour. Technol. 244: 635-640. http://dx.doi.org/10.1016/j.biortech.2017.08.010

Sun Y, Zhang ZZ, Sun YM, Yang GX (2020) One-pot pyrolysis route to Fe-N-Doped carbon nanosheets with outstanding electrochemical performance as cathode materials for microbial fuel cell. Int. J. Agric. \& Biol. Eng. 13: 207-214. https://doi.org/10.25165/j.ijabe.20201306.5765

Wang H, Li JJ, Lu YC, Guo P, Wang XF (2013) Bioconversion of un-pretreated lignocellulosic materials by a microbial consortium XDC-2. Bioresour. Technol. 136: 481-487.

https://doi.org/10.1016/j.biortech.2013.03.015

Wang J, Liu Z, Xia J, Chen Y (2019a) Effect of microbial inoculation on physicochemical properties and bacterial community structure of citrus peel composting. Bioresour. Technol. 291: 121843.

https://doi.org/10.1016/j.biortech.2019.121843

Wang S, Ma F, Ma W, Wang P, Zhao G, Lu X (2019) Influence of Temperature on Biogas Production Efficiency and Microbial Community in a Two-Phase Anaerobic Digestion System. J. Water. 11: 133. https://doi.org/10.3390/w11010133

Wang W, Yan L, Cui Z (2011) Characterization of a microbial consortium capable of degrading lignocellulose. Bioresour. Technol. 102: 9321-9324. https://doi.org/10.1016/j.biortech.2011.07.065 
Wang Z, Cheng Q, Liu Z, Qu J, Chu X, Li N, Noor RS, Liu C, Qu B, Sun Y (2019b) Evaluation of methane production and energy conversion from corn stalk using furfural wastewater pretreatment for whole slurry anaerobic codigestion. Bioresour. Technol. 293: 121962. https://doi.org/10.1016/j.biortech.2019.121962

Ward L, Taylor MW, Power JF, Scott BJ, McDonald IR, Stott M B (2017). Microbial community dynamics in Inferno Crater Lake, a thermally fluctuating geothermal spring. The ISME J. 11: 1158-1167. https://doi.org/10.1038/ismej.2016.193

Werner JJ, Knights D, Garcia ML, Scalfone NB, Smith S, Yarasheski K, Cummings TA, Beers AR, Knight R, Angenent LT (2011) Bacterial community structures are unique and resilient in full-scale bioenergy systems. Proc. Natl. Acad. Sci. U.S.A. 108: 4158-4163. https://doi.org/10.1073/pnas.1015676108

Wu L, Li H, Cao B, Zhao T, Wang Z (2021) Study on gas characterization and fluorescence characteristics of intermediates in biogenic gas production from lignite by ultrasound assisted hydrogen peroxide pretreatment. Arab J. Geosci. 14: 296. https://doi.org/10.1007/s12517-021-06676-5

Yin S, Wang XJ, Ma GY (2016) Biodegradation of corn stover by composite microbial agents. J. Fujian J. of Agricul. Sci. 31: 532-537.

Zhang L, Loh, KC, Zhang J (2018) Enhanced biogas production from anaerobic digestion of solid organic wastes: Current status and prospects. Bioresour. Technol. 5: 280-296.

https://doi.org/10.1016/j.biteb.2018.07.005

Zhang M, Riaz M, Liu B, Xia H, El-desouki Z, Jiang C (2020) Two-year study of biochar: Achieving excellent capability of potassium supply via alter clay mineral composition and potassium-dissolving bacteria activity. Sci. of The Total Environ. 717(2020): 137286. https://doi.org/10.1016/j.scitotenv.2020.137286

Zheng G, Zhao X, Li J (2017) Optimization of culture conditions and enzyme production analysis of a group of low temperature resistant cellulose degradation strains. J. Journal of Northeast Agricultural University. 4.

Zheng G, Yin T, Lu Z, Boboua SYB, Li J, Zhou W (2020) Degradation of rice straw at low temperature using a novel microbial consortium LTF-27 with efficient ability. Bioresour. Technol. 304: 123064.

https://doi.org/10.1016/j.biortech.2020.123064

\section{Figures}




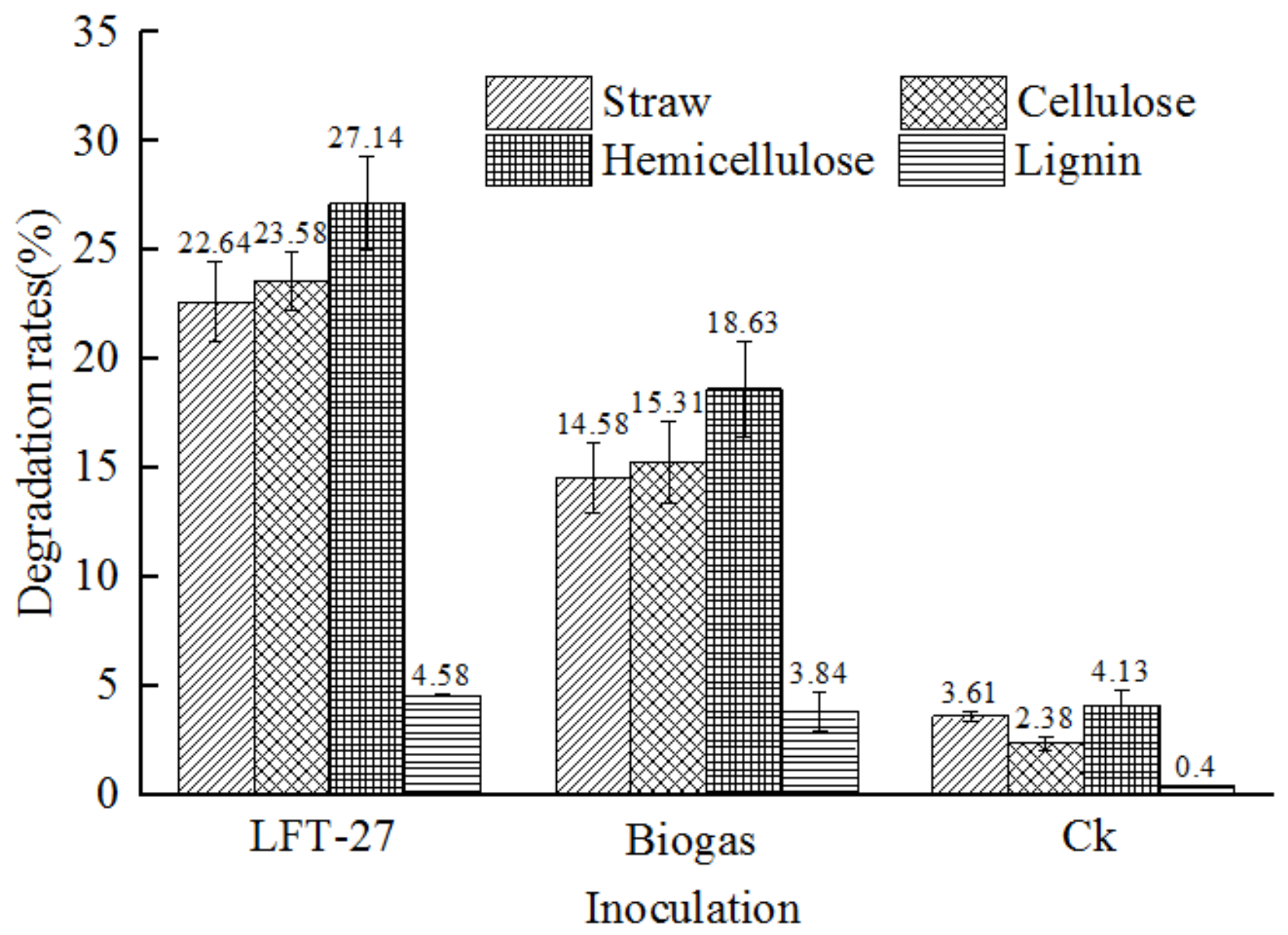

Figure 1

Degradation rates of various straw components in the hydrolysis stage, $\mathrm{CK}=$ blank control group 


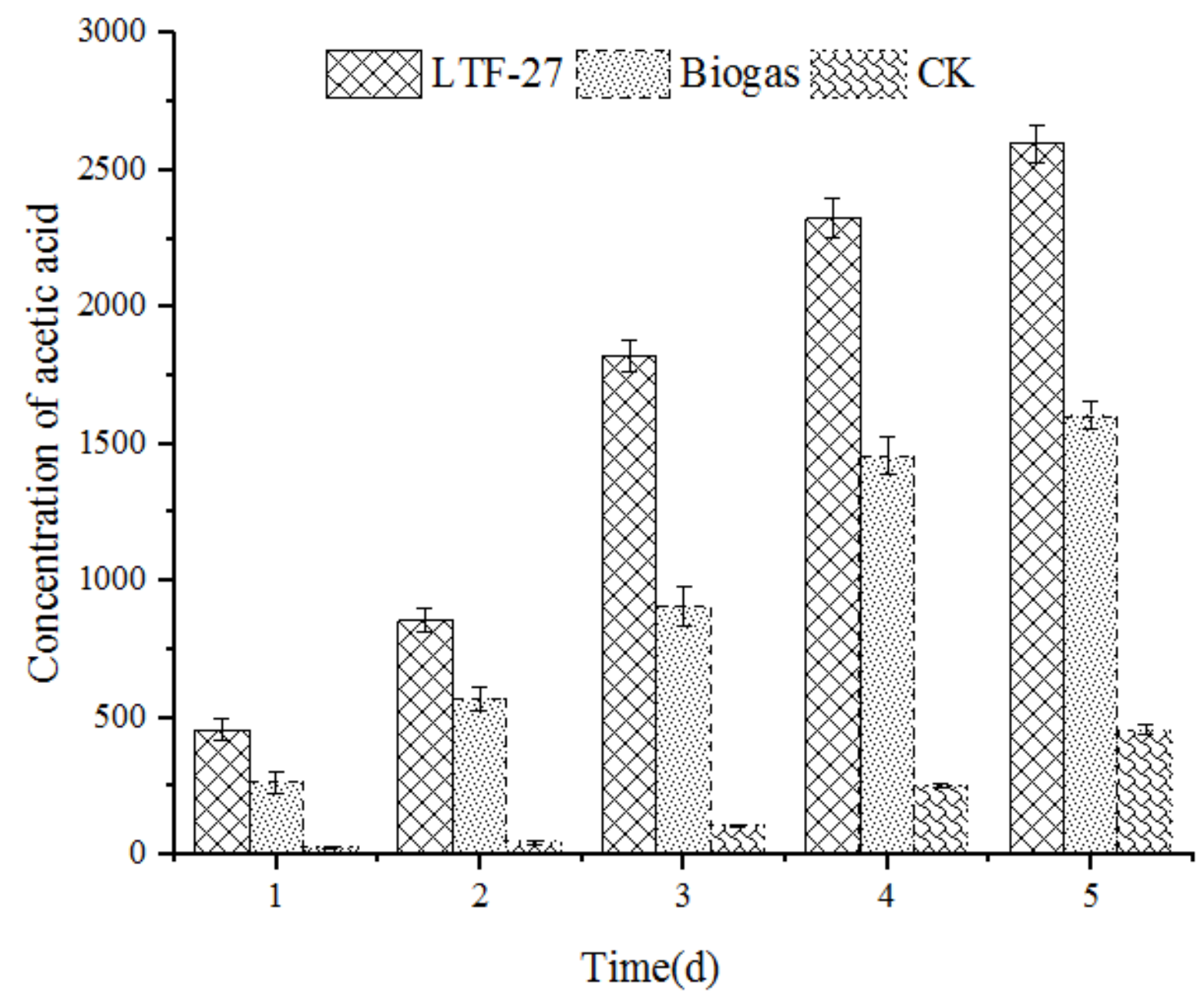

Figure 2

Changes in acetic acid concentration during hydrolysis, $\mathrm{CK}=$ blank control group 


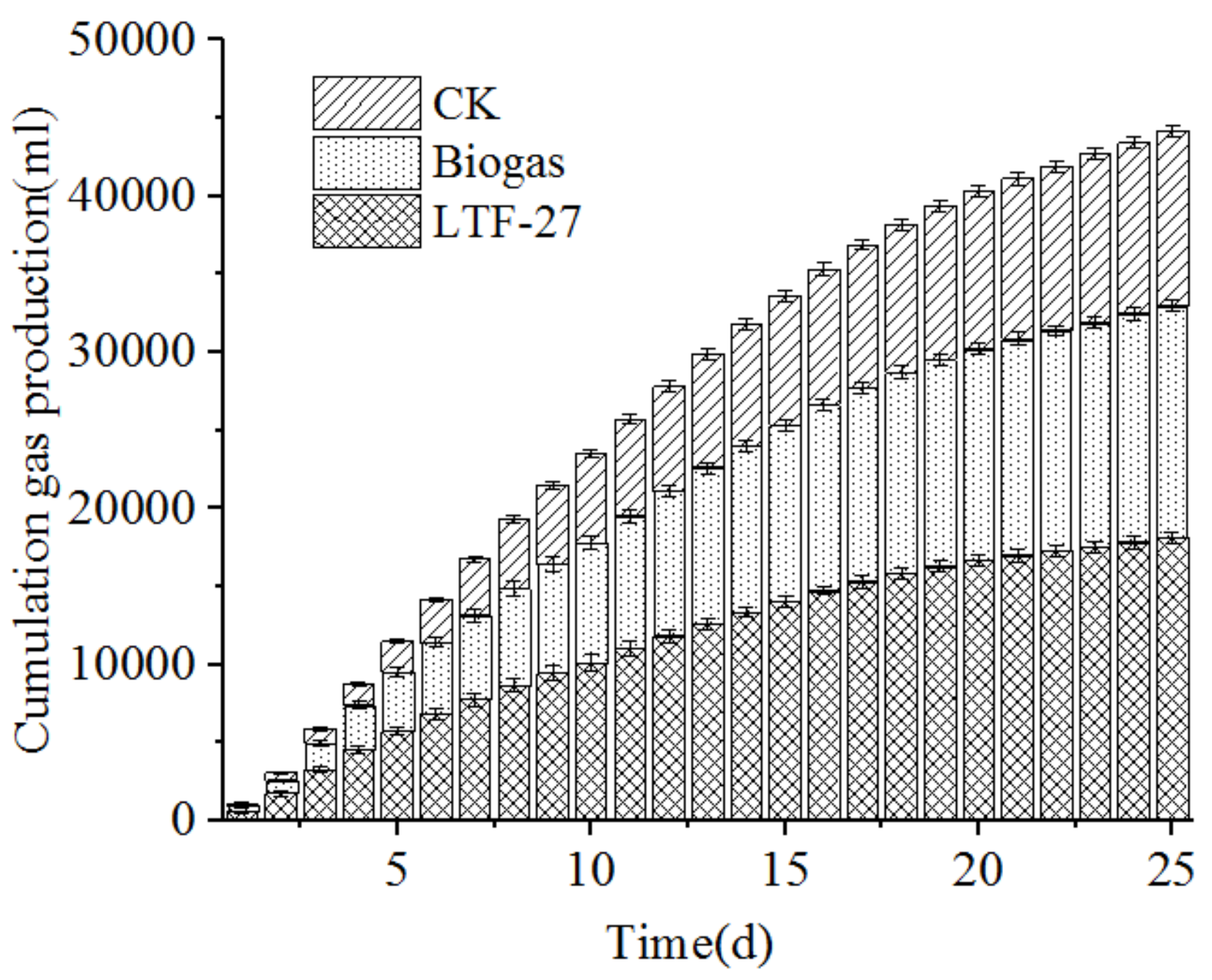

Figure 3

The effect of adding complex constraints on the cumulative system gas-production, the $\mathrm{CK}=$ blank control group 


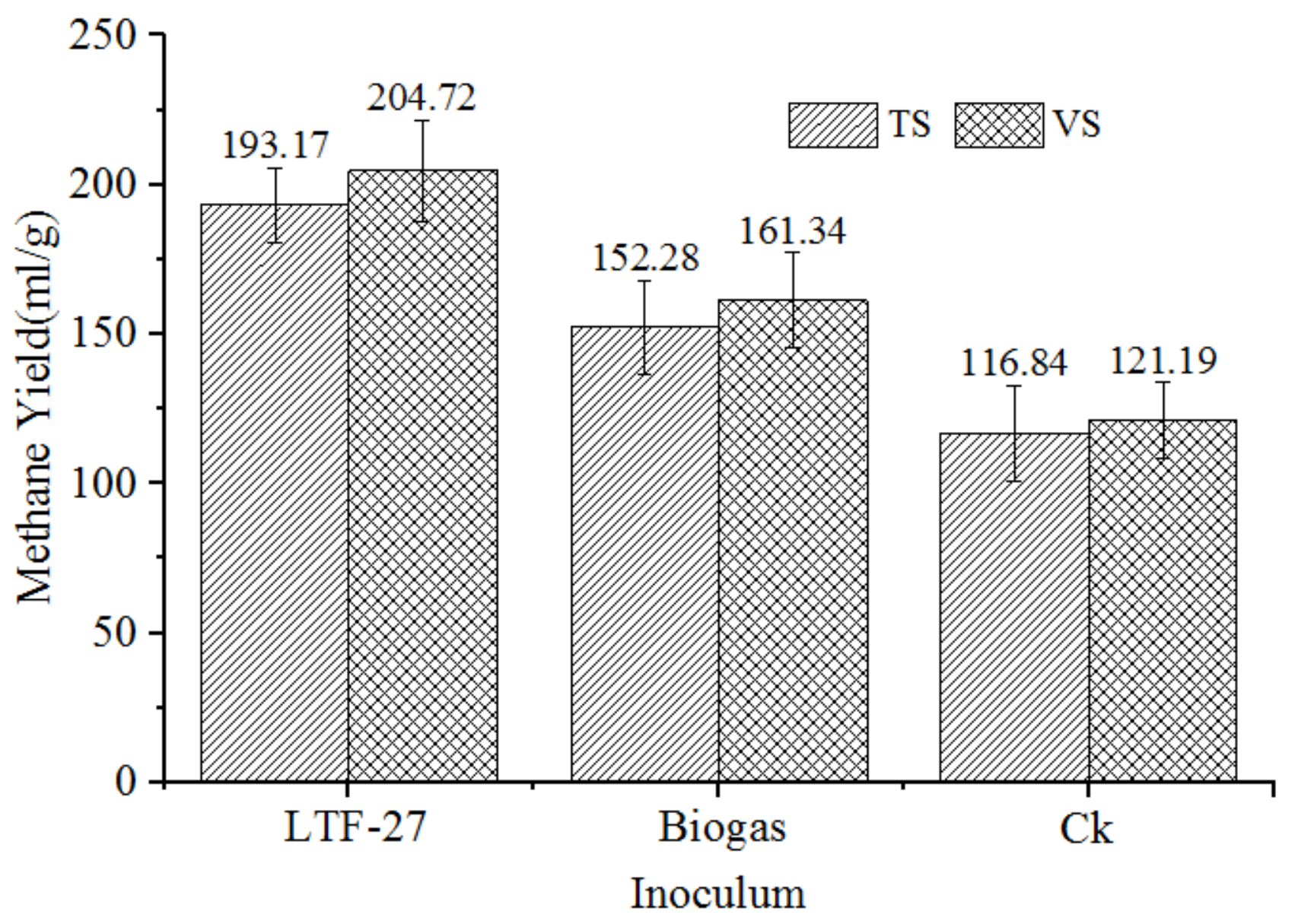

Figure 4

The effect of microbial consortium LTF-27 on methane yield in the reaction system, the CK= blank control group 


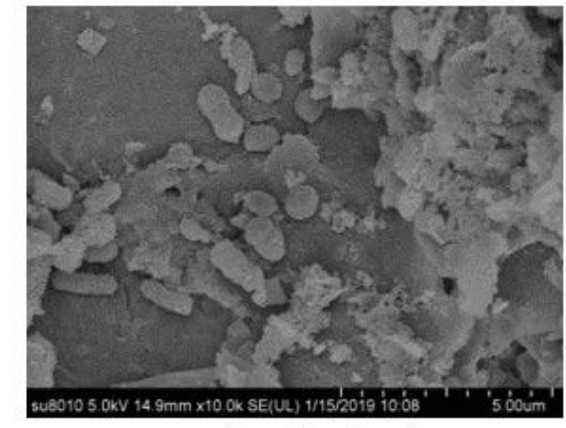

Pre-hydrolysis

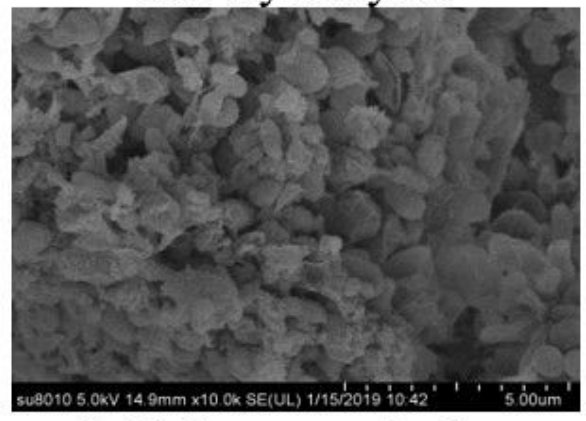

Initial gas-producing

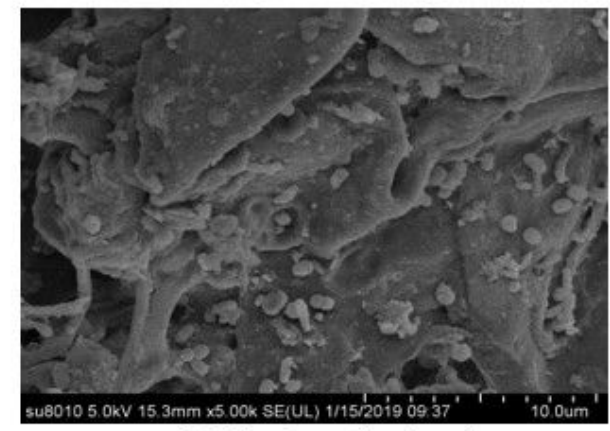

Middle hydrolysis

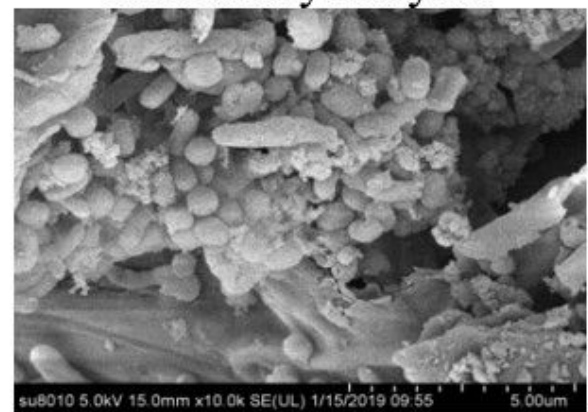

Middle gas-producing
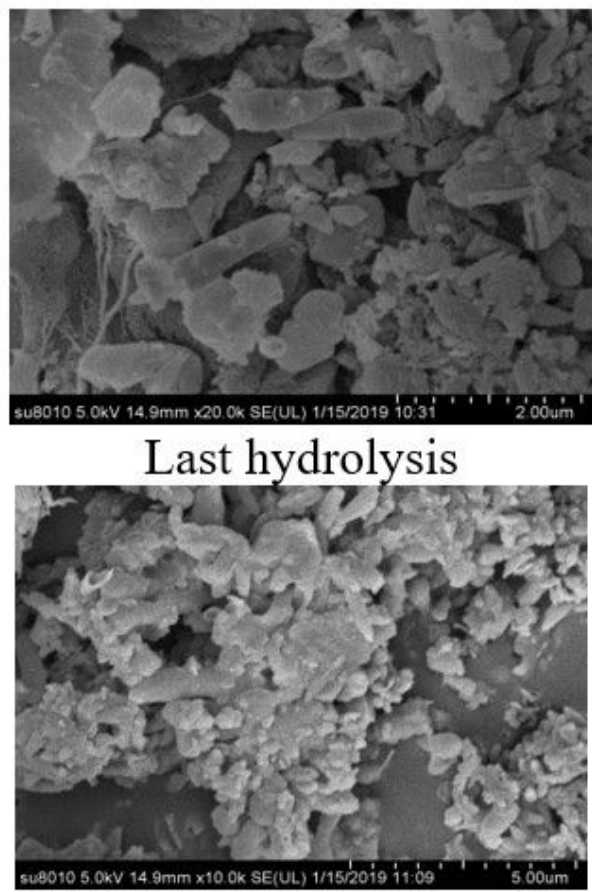

Last gas-producing

Figure 5

Morphological characteristics of microbial communities LTF-27 in a two-phase anaerobic fermentation system
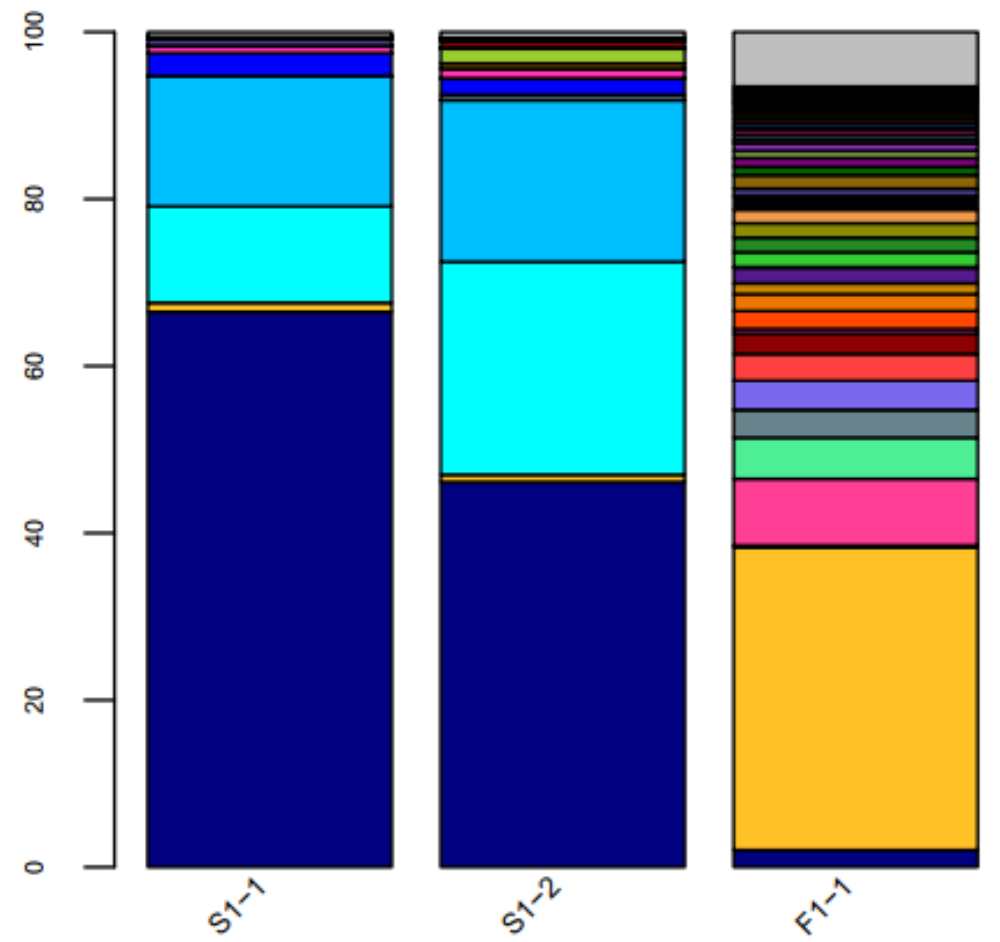

- Citrobacter

ㅁ unclassified

ㅁ Citrobacter

ㅁ Lysinibacillus

ㅁ Macellibacteroides

口 Clostridium III

ㅁ Clostridium sensu stricto

- Camobacterium

ㅁ Ruminococcus

ㅁ Cloacibacillus

- Prevotella

Clostridium $\mathrm{XJVa}$

ㅁ Proteiniphilum

ㅁ Petrimonas

ㅁ Oscillibacter

- Syntrophomonas

ㅁ Clostridium XVV

ㅁ Clostridium $I V$

ㅁ Intestinimonas

ㅁ Sedimentibacter

ㅁ Sphaerochaeta

- Pyramidobacter

口 Lysinibacillus

ㅁ Bacteroides

- Sporobacter
- Paraprevotella

ㅁ Thanera

口 Parabacteroides

- Robinsoniella

- Clostridium XI

- Acetanaerobacterium

- Phascolarctobacterium

- Romboutsia

ㅁ Lutispora

- Subdinision3_genera_incertae_sedis

ㅁ. Anaerovorax

ㅁ. Anaerorhabdus

ㅁ Luteimonas

- Christensenella

ㅁ Hydrogenispora

- Ochrobactrum

ㅁ. Alloprevotella

ㅁ Candidatus Cloacamonas

ㅁ Catabacter

- Subdivision 5 genera_incertae_sedis

ㅁ. Methanocorpusculum

口 Gracilibacter

ㅁ Bacillus

ㅁ Treponema

ㅁother

\section{Figure 6}

The relative abundance of microbial communities at the genus level 


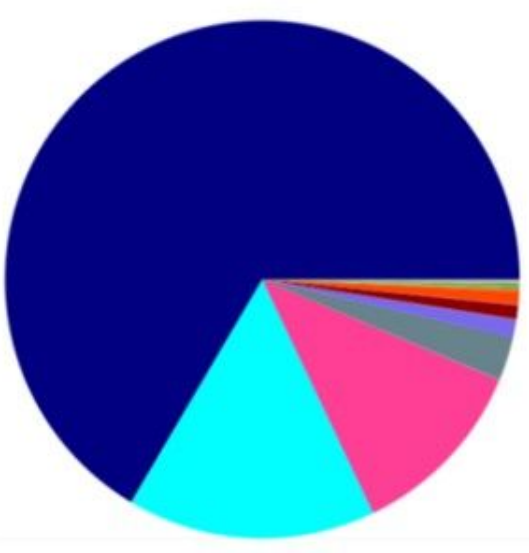

A. Bacteria-genus

(3d)

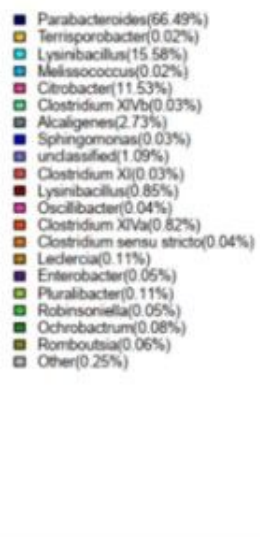

\section{Figure 7}

The relative abundance of microbial communities in the hydrolysis stage at the genus level.

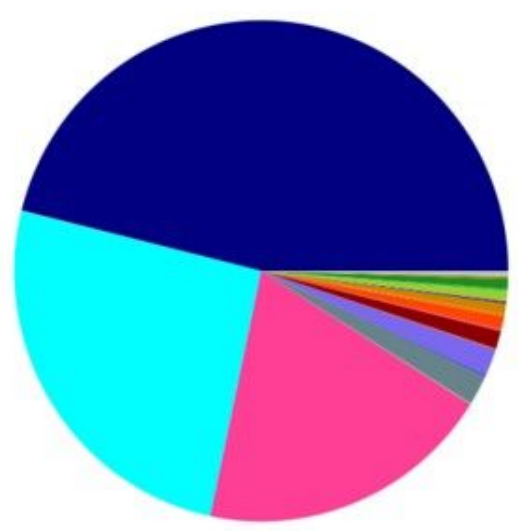

B. Bacteria-genus

$(5 \mathrm{~d})$

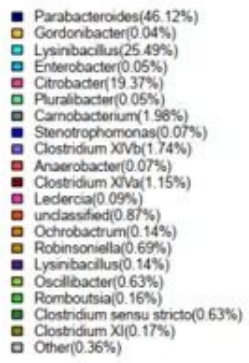

A. Bacteria-genus

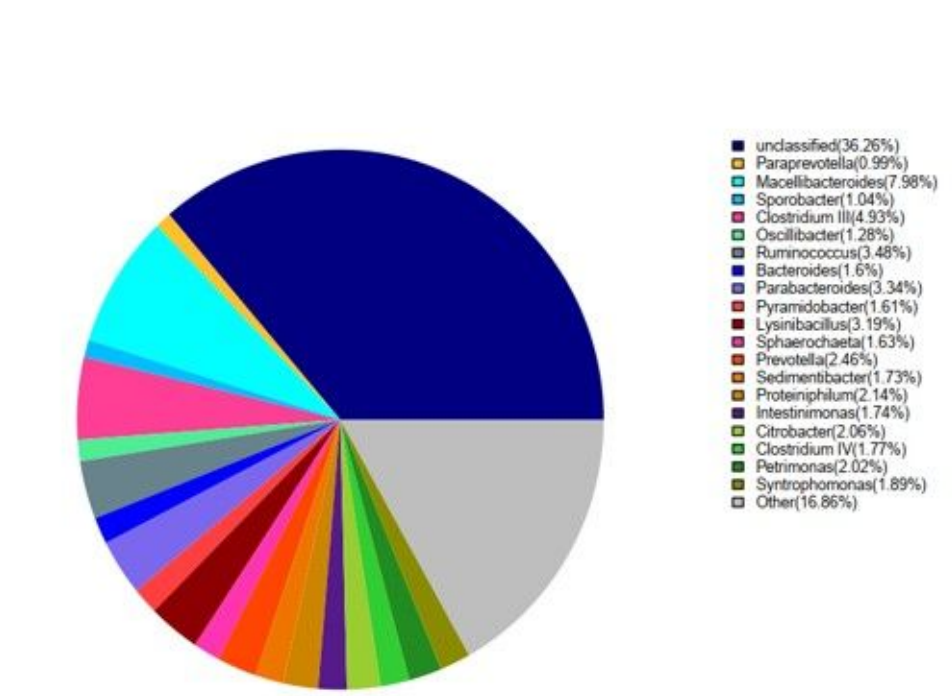

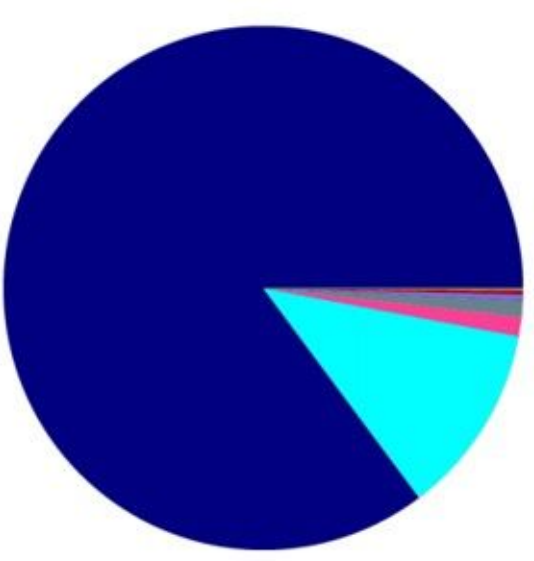

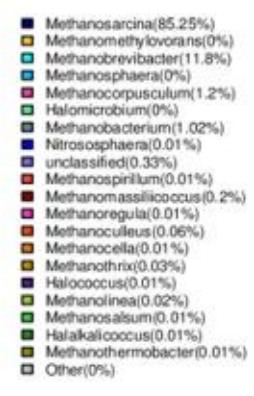

B. Archaea-genus

\section{Figure 8}

The microbial community structure in the gas-producing phase 